\title{
Respiratory muscle training in Duchenne muscular dystrophy
}

\author{
E RODILLO, ${ }^{*} \mathrm{C}$ M NOBLE-JAMIESON, ${ }^{*} \mathrm{~V}$ ABER, $\dagger \mathrm{J}$ Z HECKMATT, ${ }^{*}$ F MUNTONI, \\ AND V DUBOWITZ*
}

${ }^{*}$ Department of Paediatrics and Neonatal Medicine, and †Department of Medical Statistics, Hammersmith Hospital, London

\begin{abstract}
SUmmarY Twenty two boys with Duchenne muscular dystrophy were entered into a randomised double blind crossover trial to compare respiratory muscle training with a Triflow II inspirometer and 'placebo' training with a mini peak flow meter. Supine posture was associated with significantly impaired lung function, but respiratory muscle training showed no benefit.
\end{abstract}

The fundamental respiratory problem in Duchenne muscular dystrophy is progressive intercostal and diaphragmatic muscle weakness often complicated by scoliosis. In practice the absolute lung volumes and forced expiratory flow measurements increase up to the age of 10-12 years, after which they fail to improve further with age, producing a fall in spirometric indices in late teenage life with deterioration in blood gas concentrations and eventual death. ${ }^{1}$

One potential method of improving lung function in Duchenne muscular dystrophy is respiratory muscle training. Such a method would be useful as a means of preparing the child for surgery. Martin et $a l^{2}$ and Houser $e t a l^{3}$ have claimed benefit from respiratory muscle training, but neither used a control period of placebo training. We performed a double blind, placebo controlled, crossover study to evaluate the effect of short term respiratory muscle training on lung function.

\section{Patients and methods}

Twenty two boys with Duchenne muscular dystrophy aged 9-14 years (mean 11.6) were recruited from two special schools where they received daily physiotherapy. They were randomised to receive either respiratory muscle training using a Triflow II Inspirometer (Cheseborough Ponds) to a total of 20 inspirations/day, or 'placebo' training using a mini peak flow meter to a total of 10 expirations/day, administered by the school physiotherapists. Triflow training entailed forced inspiration against a resistance that increased as inspiratory flow increased. Preliminary studies with other children with
Duchenne muscular dystrophy showed that 20 inspirations exercised the respiratory muscles to the point of fatigue, whereas 10 forced expirations through a mini peak flow meter caused no fatigue. After 18 days using either Triflow or placebo, each child 'crossed over' to the other form of treatment for a further 18 days. An arbitrary six point scale was attached to each Triflow device so that the child's effort could be recorded. The supervising physiotherapist kept a detailed diary of the Triflow scores and the peak expiratory flow rates to ensure that each child did a comparable amount of exercise, and to look for improvements during training.

Lung function was assessed initially and at the end of each training period by two of the authors (ER, FM) who were unaware of the nature of the randomisation. Forced vital capacity (FVC), forced expiratory volume in one second $\left(F E V_{1}\right)$ were recorded, and peak expiratory flow rate (PEFR) was measured with a pocket spirometer (Micromedical). These measurements were made in each of three positions: sitting, supine, and sitting again, to assess the effects of posture and fatigue on lung function. The best of each set of three measurements in each position was used for analysis. Predicted normal values were based on armspan. ${ }^{4}$ Maximal inspiratory mouth pressure (PImax) was measured by a standard method, ${ }^{5}$ and the best of three measurements used for analysis.

To compare the effect of Triflow and placebo training we applied a standard crossover analysis that permits the treatment to be compared by paired $t$ test among all subjects if the order of administration does not alter the treatment effect. ${ }^{6}$

\section{Results}

Of the 22 children recruited to the study two were withdrawn because of illness during the study period. Eleven children started with 'placebo' respiratory muscle training using the mini peak flow meter, and crossed over to the Triflow inspirometer during the second three week period (group 1). Nine children performed the training in the reverse order (group 2). There was a significant fall in FVC when spirometry was performed in the supine position 
(mean FVC $34.6 \%$ of predicted values) compared with sitting (mean FVC $38 \%$ of predicted values, $\mathrm{p}<0.05$ ), but no significant change in any spirometric measurement between the two tests performed in the sitting position, indicating that the subjects were not fatigued during testing.

The scores obtained during the training periods using the mini peak flow meter and the Triflow inspirometer often improved during the first two or three days of training, but overall there were no significant improvements in scores during either of the training periods in both group 1 and group 2 children.

The results of the lung function tests before and after the training periods are given in table 1 . There were no significant changes in FVC or FEV during $_{1}$ either placebo training or Triflow training for either group of children when compared with baseline data. Group 1 children (who received placebo training before Triflow) showed significant improvements in PEFR during the placebo training period $(p=0 \cdot 02)$, but not during Triflow training. The same tests used to compare baseline data with Triflow training showed a significant change in PImax values $(p=0 \cdot 02)$, which suggests a strong learning effect during the study. The standard analysis for crossover designs for determining the effect of Triflow compared with placebo training showed no significant differences for any of the measurements (table 2 , column 4$),{ }^{6}$ nor did the order in which the treatments were given affect the results (table 2, column 3).

\section{Discussion}

Considering the design of this study we presume that the Triflow Inspirometer exercises the diaphragm, which is the most important muscle of respiration,

Table 1 Mean (SD) lung function tests measured in the sitting position before and after respiratory muscle training periods

\begin{tabular}{|c|c|c|c|c|c|c|c|}
\hline \multicolumn{4}{|c|}{ Group $1(n=11)$} & \multicolumn{4}{|c|}{ Group $2(n=9)$} \\
\hline FVC & $F E V_{1}$ & PEFR & PImax & $F V C$ & $F E V_{1}$ & PEFR & PImax \\
\hline \multicolumn{4}{|c|}{ Baseline measurement } & \multicolumn{4}{|c|}{ Baseline measurement } \\
\hline $1 \cdot 30(0 \cdot 51)$ & $1.20(0.46)$ & $163^{*}(65)$ & $49(21)$ & $1.58(0.53)$ & $1 \cdot 50(0 \cdot 53)$ & $209(75)$ & $43+(21)$ \\
\hline \multicolumn{4}{|c|}{ Period 1 (after placebo training) } & \multicolumn{4}{|c|}{ Period 2 (after Triflow training) } \\
\hline $1.34(0.60)$ & $1.29(0.56)$ & $194^{*}(69)$ & $60(29)$ & $1.50(0.48)$ & $1.47(0.48)$ & $226(59)$ & $62 \dagger(16)$ \\
\hline \multicolumn{4}{|c|}{ Period 2 (after Triflow training) } & \multicolumn{4}{|c|}{ Period 2 (after placebo training) } \\
\hline $1 \cdot 31(0 \cdot 55)$ & $1.24(0.49)$ & $188(76)$ & $61(24)$ & $1.58(0.53)$ & $1 \cdot 55(0 \cdot 51)$ & $240(60)$ & $75(23)$ \\
\hline
\end{tabular}

* and $\dagger: \mathrm{p}=0 \cdot 02$.

Table 2 Mean measurements of lung function measured in the sitting position, during either placebo training or respiratory muscle training using a Triflow inspirometer, to show the effect of treatment

$\begin{array}{llll}\begin{array}{l}\text { After placebo } \\ \text { training }\end{array} & \text { After Triflow } & \text { Difference } & \text { Mean (SE) } \\ & \text { training } & \text { (placebo- } & \text { Triflow } \\ & & \text { Triflow) for } & \text { effect for } \\ & & \text { each group } & \text { all 20 patients }\end{array}$

\begin{tabular}{|c|c|c|c|c|}
\hline $\begin{array}{l}\text { FVC } \\
\text { Placebo training first }(n=11) \\
\text { Triflow training first }(n=9)\end{array}$ & $\begin{array}{l}1.34 \\
1.58\end{array}$ & $\begin{array}{l}1 \cdot 31 \\
1.50\end{array}$ & $\begin{array}{l}0 \cdot 03 \\
0 \cdot 08\end{array}$ & $0.05(0.07)$ \\
\hline $\begin{array}{l}\text { FEV } \\
\text { Placebo training first }(n=11) \\
\text { Triflow training first }(n=9)\end{array}$ & $\begin{array}{l}1.29 \\
1.55\end{array}$ & $\begin{array}{l}1.24 \\
1.47\end{array}$ & $\begin{array}{l}0 \cdot 05 \\
0 \cdot 08\end{array}$ & $0.06(0.07)$ \\
\hline $\begin{array}{l}\text { PEFR }(1 / \min ) \\
\text { Placebo training first }(n=11) \\
\text { Triflow training first }(n=9)\end{array}$ & $\begin{array}{l}194 \\
240\end{array}$ & $\begin{array}{l}188 \\
226\end{array}$ & $\begin{array}{r}6 \cdot 0 \\
14 \cdot 0\end{array}$ & $9 \cdot 6(6 \cdot 7)$ \\
\hline $\begin{array}{l}\text { PImax }\left(\mathbf{c m ~} \mathrm{H}_{2} \mathbf{O}\right) \\
\text { Placebo training first }(n=11) \\
\text { Triflow training first }(n=9)\end{array}$ & $\begin{array}{l}60 \\
75\end{array}$ & $\begin{array}{l}61 \\
62\end{array}$ & $\begin{array}{r}-1 \cdot 0 \\
13 \cdot 0\end{array}$ & $5 \cdot 3(4 \cdot 4)$ \\
\hline
\end{tabular}


whereas the peak expiratory flow meter exercises the muscles of the abdomen and other accessory muscles, which are less important. In their study of training Martin et al claimed to show improvement of muscle endurance, ${ }^{2}$ but the clinical value of this is uncertain. The vital capacity is possibly the best index of lung function as it has been repeatedly shown to predict both survival, and the ability to withstand spinal surgery without respiratory complications.

In this study we have attempted to eliminate bias in lung function testing by a double blind placebo design. When learning effects are allowed for, this study does not convincingly show any improvement in lung function from short term respiratory muscle training. It is important to note that lung function is significantly worse in the supine position-a factor likely to be relevant in postoperative care.

We thank Mrs J Perk, physiotherapist, and the pupils of the Lord Mayor Trealors School, Alton, and Mrs J Breese, physiotherapist. and pupils of Sherbourne School. Coventry, for their help and enthusiasm in completing this study. Our muscle research programme is supported by a centre grant from the Muscular Dystrophy Group of Great Britain.

\section{References \\ 1 Rideau Y, Janowski LW. Grellet J. Respiratory function in the muscular dystrophies. Muscle Nerve 1981;4:155-64. \\ ${ }^{2}$ Martin AJ, Stern L. Yeates J, Lepp D, Little J. Respiratory muscle training in Duchenne muscular dystrophy. Dev Med Child Neurol 1986:28:314-8. \\ ${ }^{3}$ Houser CR, Johnson DM. Breathing exercises for children with pseudohypertrophic muscular dystrophy. Phys Ther 1971:51: 751-9. \\ ${ }^{4}$ Godfrey F, Kamburoff PL, Nairn JR. Spirometry, lung volumes and airway resistance in normal children aged 5-18 years. $\mathrm{Br} \mathrm{J}$ Dis Chest 1970;64:15-24. \\ 5 Gaultier C, Zinman R. Maximal static pressures in healthy children. Respir Physiol 1983;51:45-61. \\ ${ }^{6}$ Mills M, Armitage P. The two-period cross-over clinical trial. $\mathrm{Br}$ J Clin Pharmacol 1979;8:7-20.}

Correspondence to Professor V Dubowitz, Department of Paediatrics and Neonatal Medicine. Hammersmith Hospital, London W12 OHS.

Accepted 14 November 1988

\title{
Congenital tuberculosis localised to the ear
}

\author{
R C NARANBHAI, W MATHIASSEN, AND A F MALAN \\ Department of Paediatrics, University of Cape Town, South Africa
}

\begin{abstract}
SUMmarY We report two infants who had localised congenital tuberculous otitis. In both cases the infants presented with an ear discharge and both mothers had been diagnosed as having miliary tuberculosis. Infection is thought to have occurred in utero or during birth.
\end{abstract}

Congenital tuberculosis is seldom reported, and when it occurs it tends to be a multisystem disorder with a poor perinatal outcome..$^{1-3}$ In older children isolated tuberculous otitis is well described ${ }^{4}$; isolated congenital tuberculosis of the ear, however, has not been previously reported.

\section{Case reports}

CASE 1

A boy, weighing $670 \mathrm{~g}$, was born vaginally at 27 weeks' gestation. The mother, a 27 year old primigravida, had booked at 14 weeks. At 23 weeks she was admitted for minor antepartum haemorrhage and unexplained fever. There were no respiratory symptoms. Several blood and urine cultures were negative. A fluctuating temperature persisted despite treatment with paracetamol, indomethacin, and co-trimoxazole. Eight days before delivery, on radiological evidence, a diagnosis of miliary tuberculosis was made and treatment was started with isoniazid, ethambutol, rifampicin, and pyrazinamide. Despite indomethacin and hexoprenaline suppression, spontaneous premature labour and delivery ensued.

Histological examination of the placenta showed small areas of infarcted villi with associated haemorrhage and fibrin deposits on maternal surfaces. Areas of caseous necrosis were noted (figure). Numerous acid-alcohol fast bacilli were seen on Ziehl-Nielsen staining. This information was, however, only discovered after discharge of the infant.

Immediately after delivery the infant was taken to the neonatal intensive care unit. He had clinical and radiological features of hyaline membrane disease, but required headbox oxygen only. Apnoeic spells occurred from days 15 to 45 . Investigations for sepsis and metabolic abnormalities were negative, 